\title{
Enzymatically prepared dual functionalized hydrogels with gelatin and heparin to facilitate cellular attachment and proliferation
}

Wahyu Ramadhan ${ }^{1}$, Genki Kagawa ${ }^{1}$ Yusei Hamada ${ }^{1}$, Kousuke Moriyama ${ }^{2}$, Rie Wakabayashi ${ }^{1}$, Kosuke Minamihata ${ }^{1}$, Masahiro Goto ${ }^{1,3}$, and Noriho Kamiya ${ }^{1,3 *}$

${ }^{1}$ Department of Applied Chemistry, Graduate School of Engineering, Kyushu University, 744 Motooka, Fukuoka 819-0395, Japan.

2 Department of Chemical and Biological Engineering, Sasebo National College of Technology, Okishin-cho, Sasebo, Nagasaki, 857-1193, Japan

${ }^{3}$ Division of Biotechnology, Center for Future Chemistry, Kyushu University, 744 Motooka, Fukuoka 819-0395, Japan.

*Corresponding author

E-mail: kamiya.noriho.367@,m.kyushu-u.ac.jp 


\section{Contents}

1. Supplementary Results S-3

1-1. Thiol measurements $\quad$ S-3

1-2. Immobilization strategies of growth factor $\quad \mathrm{S}-4$

1-3. Arginine density of gelatin $\quad \mathrm{S}-5$

1-4. Compatibility of Hepa-SH and Gela-SH in the PEG_hydrogels S-6

1-5. Swelling behavior of the PEG/Gela/Hepa_hydrogels $\quad$ S-7

1-6. Evaluation of cellular adhesion on the PEG/Gela_hydrogels $\quad$ S-8

1-7. Antiproliferative effects of native Hepa and Hepa-SH S-9

1-8. Effect of various Hepa-SH concentrations on the numbers of NIH3T3 cells $\begin{array}{ll}\text { treated with exogenous bFGF } & \text { S-10 }\end{array}$

1-9. Cell length measurements $\quad$ S-11

1-10. Measurement of the loading capacity of bFGF on PEG/Gela/Hepa_hydrogels and the release profile of bFGF from the loaded hydrogels $\quad \mathrm{S}-12$

$\begin{array}{lr}\text { 2. References } & \text { S-13 }\end{array}$ 


\section{Supplementary results}

\section{1-1. Thiol measurements}

All thiol gelatin samples were analyzed by Ellman's reagent assay. DTNB reacted with a free sulfhydryl group to yield a mixed disulfide and 2-nitro-5-thiobenzoic acid (TNB). A cysteine solution was used to estimate free thiols. Absorbance was measured with a microplate reader (Power Wave X, Bio-Tec Instruments Inc., USA) at $412 \mathrm{~nm}$.

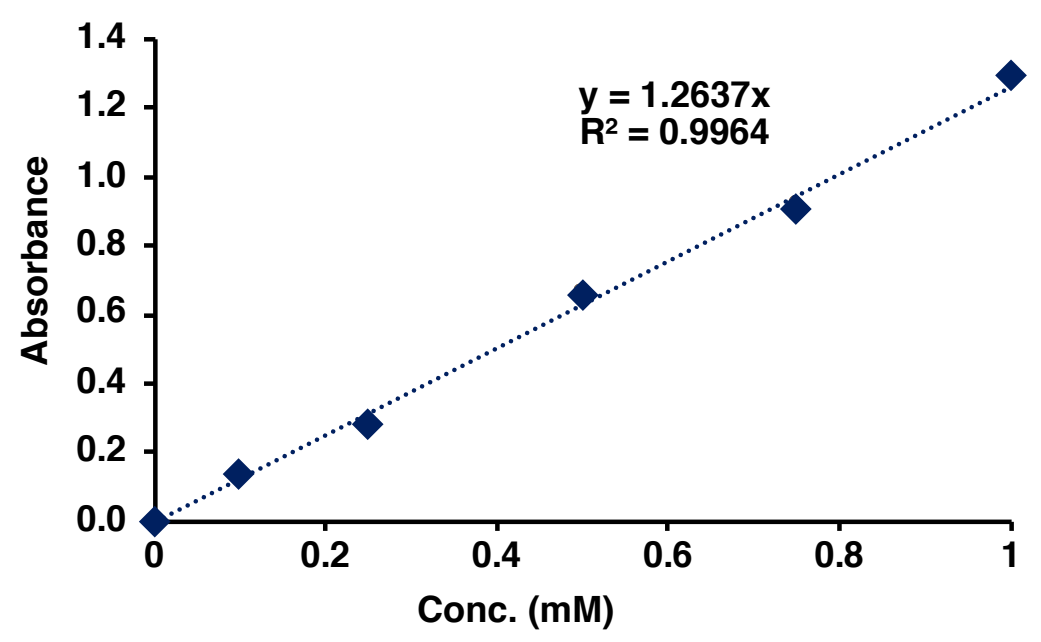

Figure S1. Calibration curve obtained by measuring the absorbance of the cysteine solution 


\section{1-2. Immobilization strategies of growth factors}

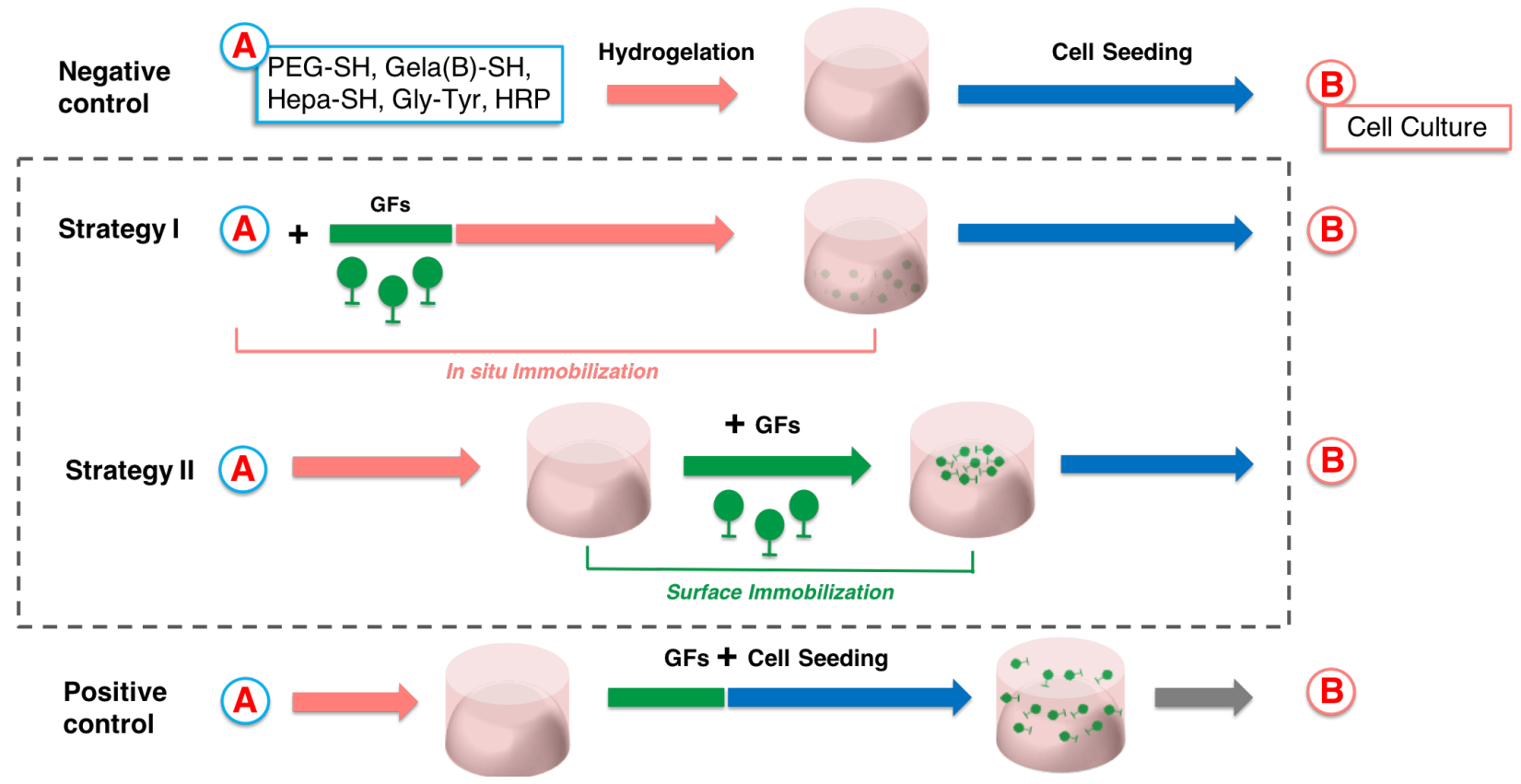

Figure S2. Schematic illustration of growth factor immobilization strategies 


\section{1-3. Arginine density of gelatin}

The amount of free arginine side chains is measured using a fluorometric technique adapted from previous studies ${ }^{1,2}$. The arginine density in Gel-SH (from bovine or porcine gelatin) was quantified by reacting arginine groups with 9,10-phenanthrenequinone to produce a fluorescent compound. Several diketo compounds, such as 2-amino- $1 H$-phenanthrol[9,10-d] imidazole and 9,10-phenanthrenequinone, form a stable fluorescent compound upon reaction with arginine. 9,10-phenanthrenequinone has been shown to react with arginine and related compounds containing quanidinium groups. Briefly, $1 \mathrm{mg} / \mathrm{mL}$ gelatin or gel-SH was mixed with $300 \mu \mathrm{L}$ of an ethanol solution of 9,10-phenanthrenequinone $(150 \mu \mathrm{M})$ and $50 \mu \mathrm{L}$ of an $\mathrm{NaOH}$ aqueous solution $(2 \mathrm{~N})$. The mixture was incubated at $60{ }^{\circ} \mathrm{C}$ in the dark for $3 \mathrm{~h}$. Then, $200 \mu \mathrm{L}$ of the gelatin solution was mixed with $200 \mu \mathrm{L} \mathrm{HCl}(1.2 \mathrm{~N})$, and the mixture was allowed to stand at room temperature in the dark for $1 \mathrm{~h}$. Fluorescence emission of the mixture was measured at 355 $\mathrm{nm}$ with an excitation wavelength of $312 \mathrm{~nm}$ by a LS-55 spectrofluorometer (Perkin Elmer, USA).

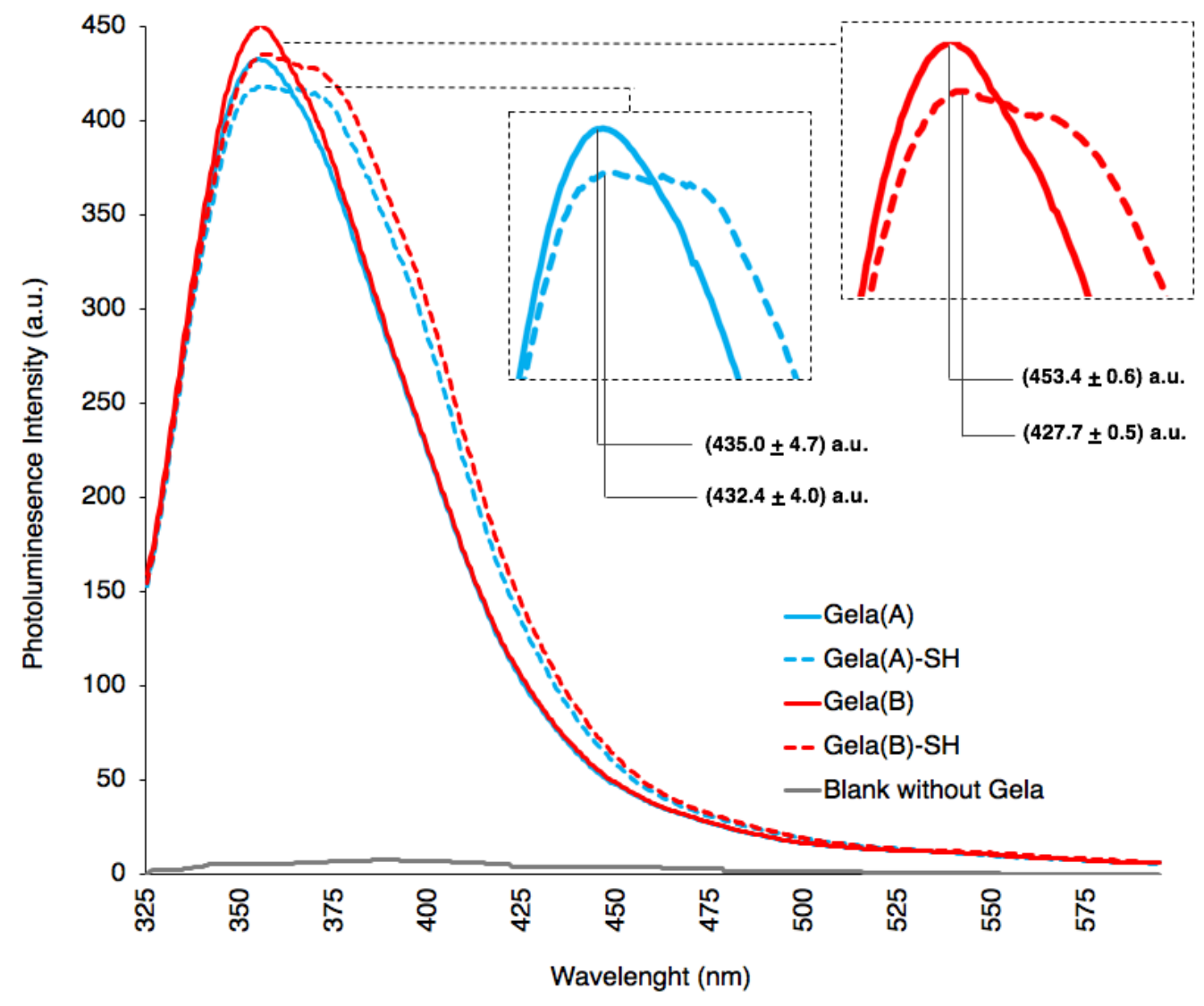

Figure S3. Arginine density-photoluminescence spectra of 9,10-phenanthrenequinone after reacting with native gelatin and thiolate gelatin. 


\section{1-4. Compatibility of Hepa-SH and Gela-SH in PEG_hydrogels}

PEG-SH, Gly-Tyr, Gela-SH, Hepa-SH, and HRP were dissolved in phosphate buffered saline (PBS). The different types of Gela-SH were Gela(A)-SH and Gela(B)-SH. The concentrations of PEG-SH, Gela-SH, Hepa-SH, Gly-Tyr, and HRP were 5\% (w/v), 0.1\% (w/v), 0.1\% (w/v), 5 mM, and $5 \mathrm{U} / \mathrm{mL}$, respectively.
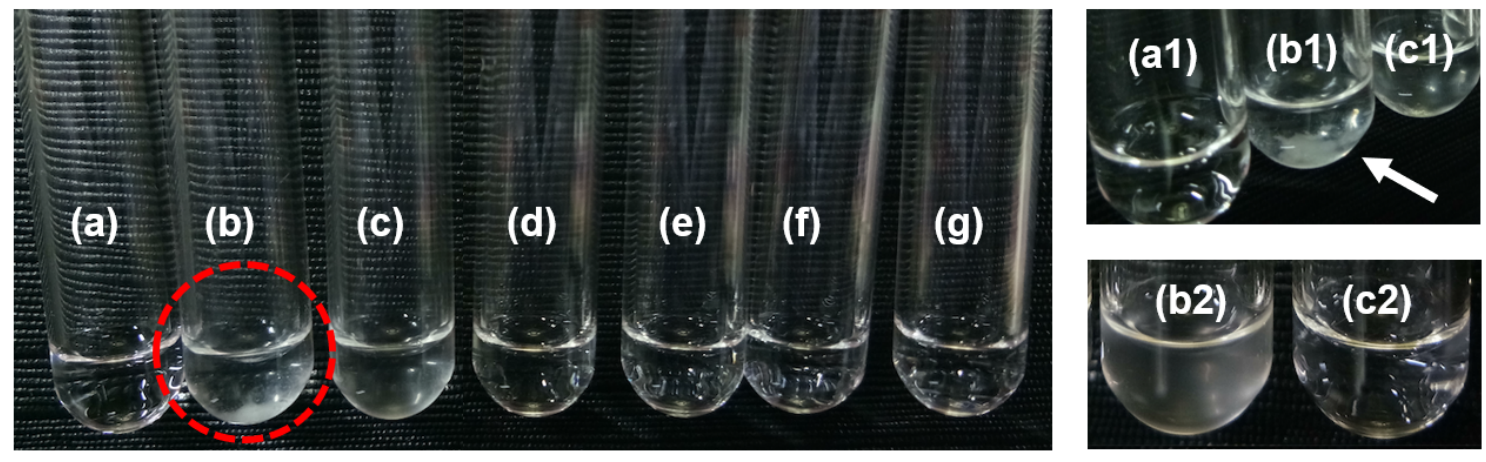

Figure S4. Compatibility charge of Hepa-SH (0.1\%) and Gela-SH (0.1\%) in the PEG-SH hydrogel system. (a; a1) PEG_hydrogel, (b; b1) PEG/Gela(A)/Hepa_hydrogel, (c; c1) PEG/Gela(B)/Hepa_hydrogel, (d) PEG/Gela(A)_hydrogel, (e) PEG/Gela(B)_hydrogel, (f) PEG/Hepa_hydrogel, and (g) PBS. Experiments were also conducted with Hepa-SH (0.1\%) and a lower Gela-SH concentration (0.01\%): (b2) PEG/Gela(A)/Hepa_hydrogel and (c2) PEG/Gela(B)/Hepa_hydrogel. 


\section{1-5 Swelling behavior of PEG/Gela/Hepa_hydrogels}

The hydrogels contained PEG-SH, Gela-SH, Gly-Tyr, and HRP at 5\% (w/v), 0.1\% (w/v), $5 \mathrm{mM}$, and $5 \mathrm{U} / \mathrm{mL}$, respectively. Gela(A)-SH and Gela(B)-SH were used. The hydrogels were shaped into a disk (diameter: $\sim 1.5 \mathrm{~cm}$; thickness: $3 \mathrm{~mm}$ ) and incubated in $10 \mathrm{~mL}$ PBS (pH 7.4) at $37^{\circ} \mathrm{C}$ for 4 days to reach equilibrium. The mass of the hydrogels was measured after swelling $\left(M_{\mathrm{S}}\right)$. The hydrogels were then dried in an oven and their dry masses $\left(M_{\mathrm{D}}\right)$ were measured. The equilibrium swelling ratio $\left(\mathrm{Q}_{\mathrm{M}}\right)$ was calculated after excluding the effect of the weight of solutes in PBS according to $\mathrm{Eq}^{3}$.

$$
\mathrm{Q}_{\mathrm{M}}=\frac{M S}{M D}
$$

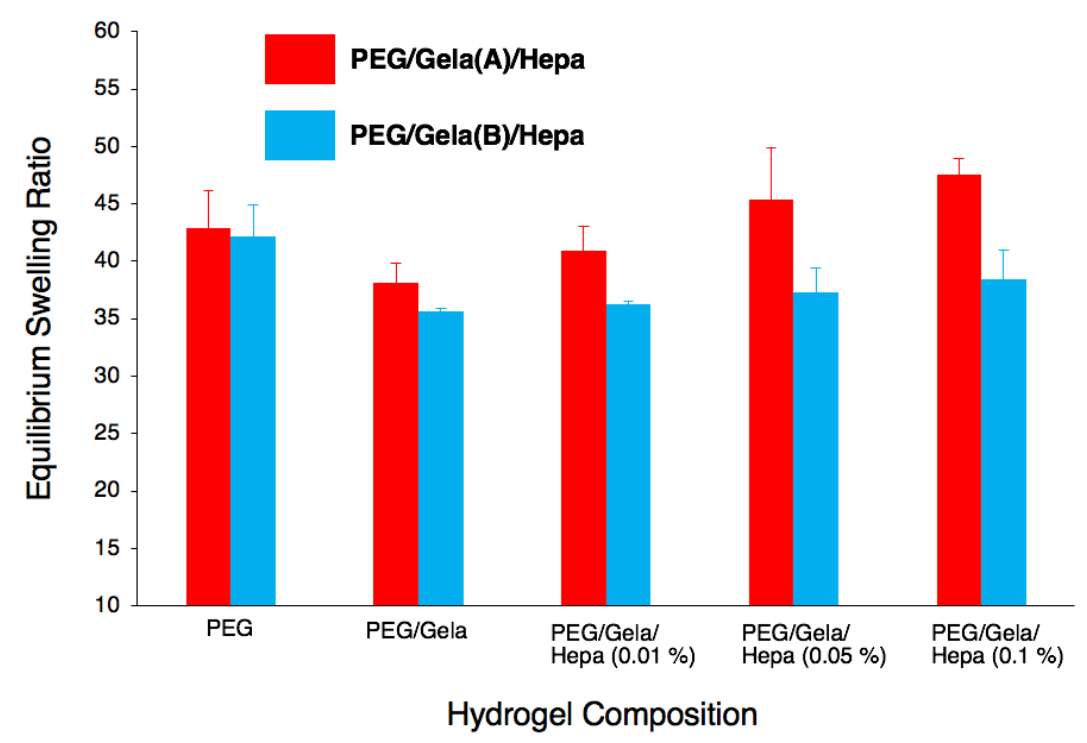

Figure S5. Swelling evaluation of PEG/Gela/Hepa_hydrogels with different Gel-SH types and Hepa-SH concentrations. 


\section{1-6. Evaluation of cellular adhesion on PEG/Gela_hydrogels}

Initial cell experiments to evaluate Gela-SH effects were conducted by seeding NIH3T3 cells (fibroblasts) on hydrogels for $6 \mathrm{~h}$. First, PEG-SH, Gly-Tyr, Gela-SH, and HRP were dissolved in phosphate buffered saline (PBS). The concentrations of PEG-SH, Gel-SH, Gly-Tyr, and HRP were $5 \%(\mathrm{w} / \mathrm{v}), 0.1 \%(\mathrm{w} / \mathrm{v}), 5 \mathrm{mM}$, and $5 \mathrm{U} / \mathrm{mL}$, respectively. Gela(A)-SH and Gela(B)-SH were used. All materials were mixed and added to a 24 -well plate. The gels were prepared at a total volume of $250 \mu \mathrm{L}$ and incubated for $6 \mathrm{~h}$. Six hours later, NIH3T3 cells were added at $1 \times 10^{5}$ cells/well. After $6 \mathrm{~h}$, cell imaging was performed under a microscope at $\times 10$ magnification.
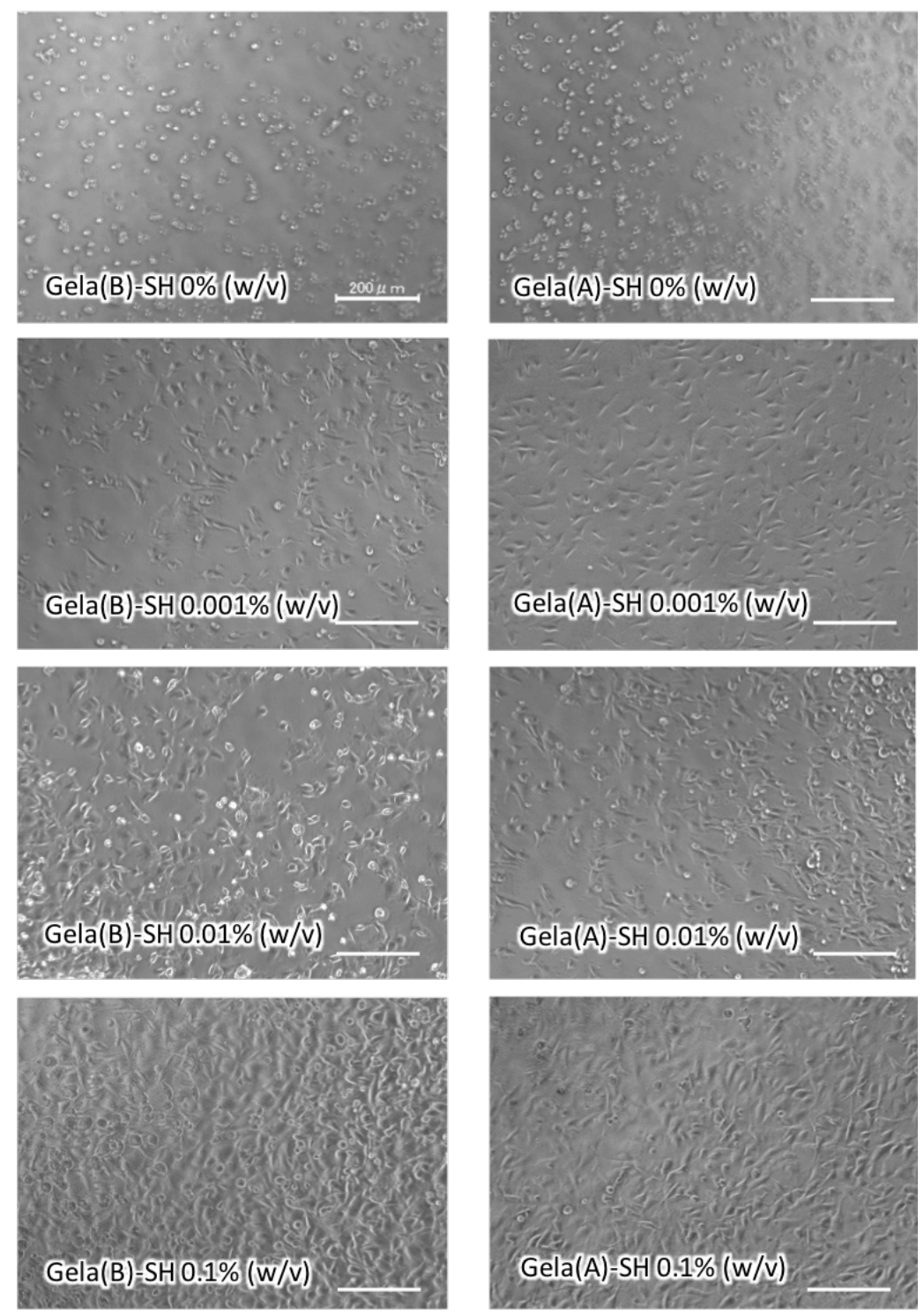

Figure S6. NIH3T3 cells adhesion evaluation after 6 h of culture on PEG/Gela_hydrogels 


\section{1-7. Antiproliferative effects of native heparin and Hepa-SH}

Heparin inhibition was evaluated by comparing fibroblast proliferation on hydrogels with different heparin concentrations by the initial cell number on a hydrogel without heparin incorporation. The hydrogels consisted of PEG-SH, Gly-Tyr, Gela(B)-SH, Hepa-SH, and HRP. The concentrations of Hepa-SH were $0.00001 \%-1 \%(w / v)(A T)$. All materials were mixed and added to a 96-well plate, followed by incubation for $6 \mathrm{~h}$. Cells were then seeded at $1 \times 10^{4}$ cells/well. Serum-free medium was used for the cell culture. Three days later, fibroblasts were counted by an automated cell counter TC 20 (Bio-Rad, Singapore) using a dual chamber counting slide and trypan blue staining (0.4\%) (Gibco, USA). Experiments were performed in triplicate. Additionally, the cell number on a hydrogel without heparin was evaluated as a control treatment (A0). The percentage of proliferation inhibition was calculated by the following equation:

$$
\% \text { Inhibition of cell proliferation }=\frac{\mathrm{A} 0-\mathrm{AT}}{\mathrm{A} 0} \times 100 \%
$$

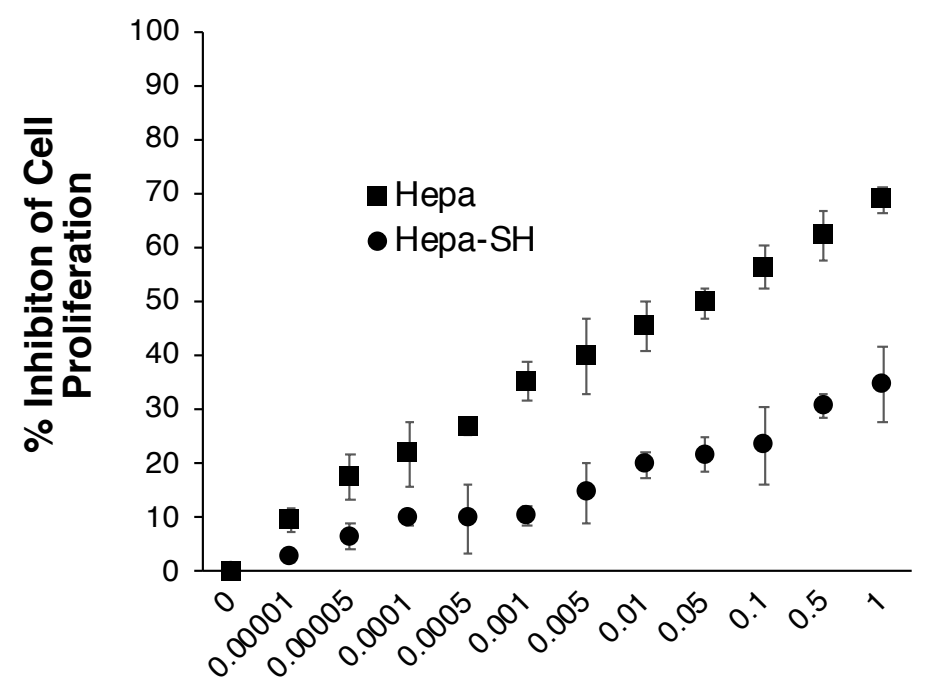

Concentration Hepa/Hepa-SH (\%)

Figure S7. Anti-proliferative effect of native heparin (Hepa) vs Hepa-SH in a PEG/Gela(B)/Hepa_hydrogel 


\section{1-8. Effect of various Hepa-SH concentrations on the numbers of NIH3T3 cells treated with exogenous bFGF}

To confirm the effect of the different Hepa-SH concentration on cell proliferation with growth factor (GF) immobilization, $0.1 \% \mathrm{Gel}(\mathrm{B})-\mathrm{SH}$ and $10 \mathrm{ng} / \mathrm{mL}$ bFGF were loaded into the hydrogel system with NIHT3T cells $\left(5 \times 10^{3} / \mathrm{cm}^{2}\right)$. The hydrogel was prepared with PEG-SH, Gly-Tyr, Gela-SH, Hepa-SH, and HRP. The concentration of Hepa-SH ranged from $0.00001 \%$ to $1 \%$ $(\mathrm{w} / \mathrm{v})$.

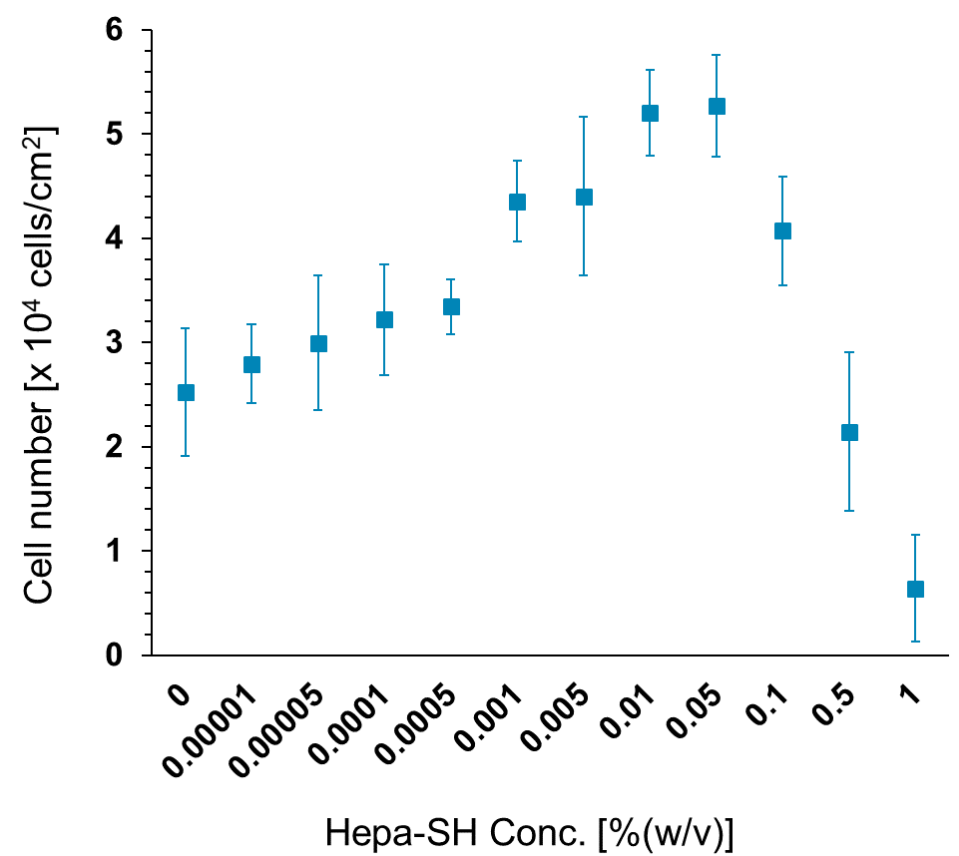

Figure S8. Effect of various Hepa-SH concentrations on the numbers of NIH3T3 cells treated with exogenous bFGF $(10 \mathrm{ng} / \mathrm{mL})$. Cells were seeded on a PEG/Gela(B)/Hepa_hydrogel containing 5\% PEG-SH, 0.1\% Gel(B)-SH, and various concentrations of Hepa-SH. Error bars denote standard deviation $(N=3)$. 


\section{1-9. Cell length measurement}

The cell length was defined as the length of a direct line between two points at the maximal distance on the outline of a cell by following previous studies. ${ }^{4,5}$. A representative image of a NIH-3T3 cell and the cell length measurement by using NIH Image J was shown below.
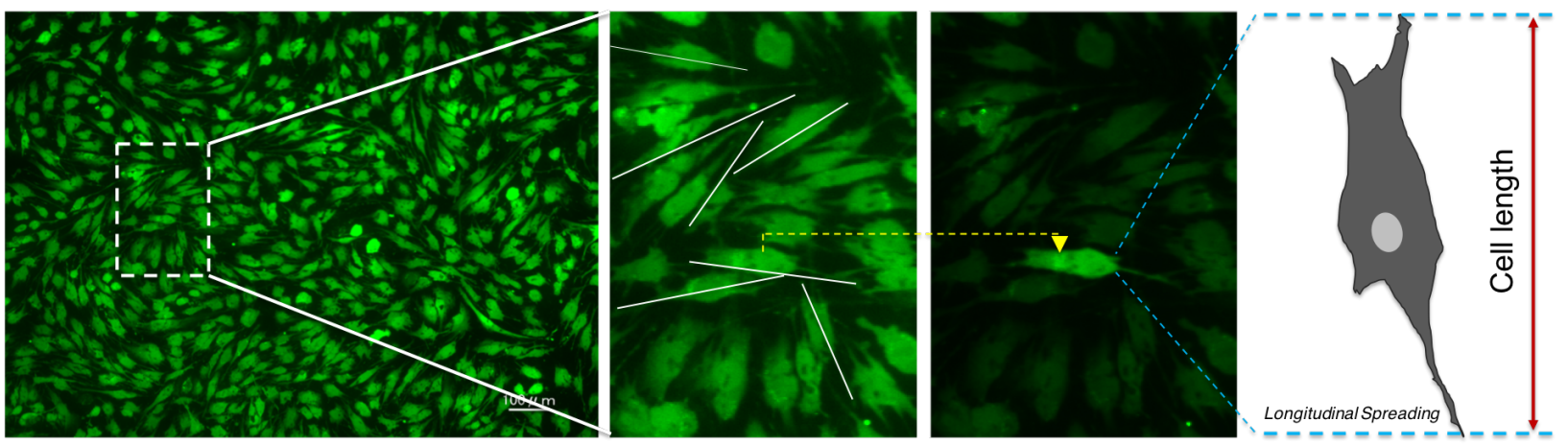

Figure S9. Outline of the determination of cell length by using a stained cell image captured by NIH Image Software. 


\section{1-10. Measurement of the loading capacity of bFGF on PEG/Gela/Hepa_hydrogels and the release profile of bFGF from the loaded hydrogels}

The amount of bFGF loaded in PEG/Gela/Hepa hydrogel was measured by ELISA according to a standard protocol provided by the manufacturer. Hydrogel was fabricated by using strategy I and II as mentioned in experimental section. Hydrogels obtained were washed twice with PBS, then an aqueous solution of $10 \mathrm{mM}$ Cys was added. After $1 \mathrm{~h}$, hydrogels were degraded and the recovered solution was used for ELISA assay. The loading capacity of bFGF (\%) was calculated by comparing the amount of bFGF in the recovered solution with the initial amount of bFGF in PEG/Gela/Hepa_hydrogel.

To check the release profile of bFGF, we measured the amount of bFGF in medium at $0,1,2,3$, $6,24,48,72,96,120,144$ and $168 \mathrm{~h}$ culture time (7 days). The amount of bFGF in medium (MEM) was compared with the initial amount of bFGF loaded in the hydrogel.
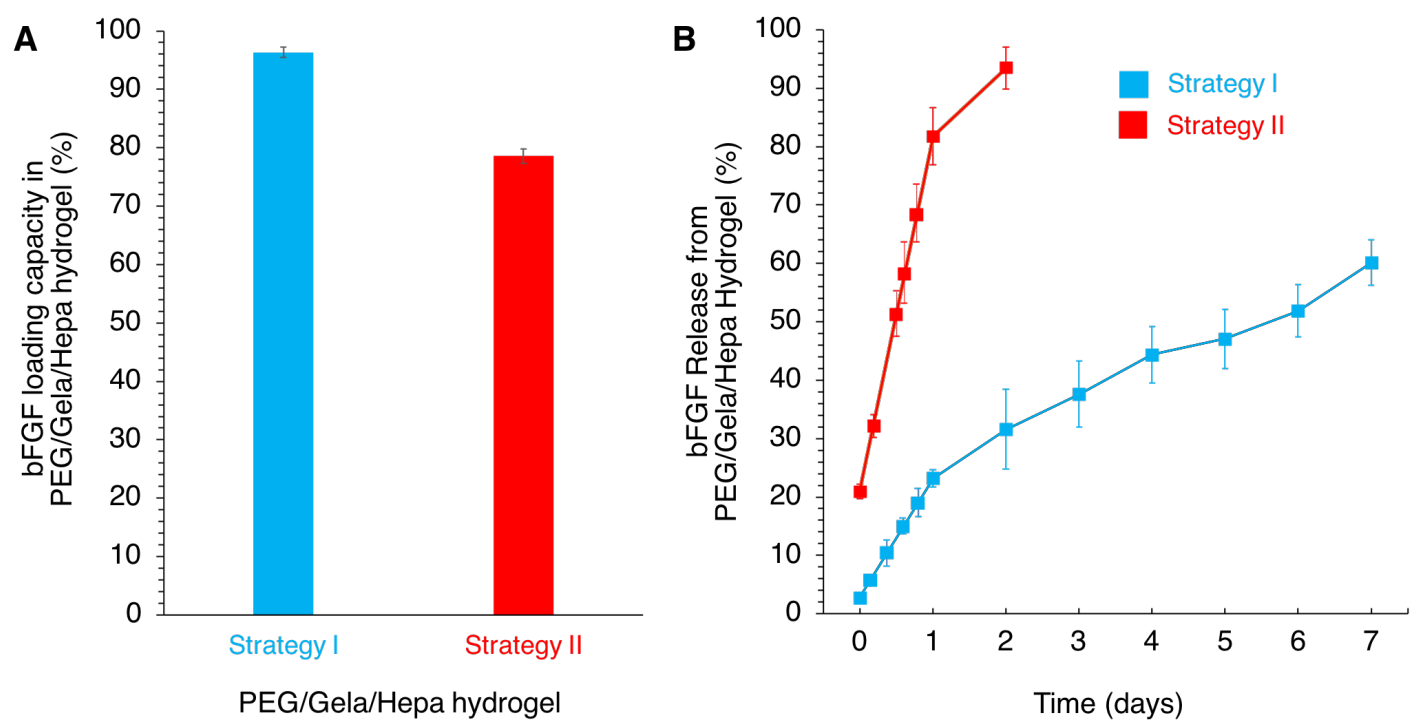

Figure S10. (A) Loading capacity of bFGF on PEG/Gela/Hepa_hydrogels prepared by strategy I and II; (B) Release profile of bFGF from PEG/Gela/Hepa_hydrogels prepared by strategy I and II. 


\section{References}

(1) Li, X.; Chen, S.; Li, J.; et al. 3D Culture of Chondrocytes in Gelatin Hydrogels with Different Stiffness. Polymers (Basel) 2016, 8.

(2) Smith, R.E.; MacQuarrie, R. A Sensitive Fluorometric Method for the Determination of Arginine Using 9,10-Phenanthrenequinone. Anal Biochem 1978, 90, 246-255.

(3) Lee, K.Y.; Mooney, D.J. Hydrogels for Tissue Engineering. Chem Rev 2001, 101, 18691880 .

(4) Levina, E.M.; Kharitonova, M.A.; Rovensky, Y.A.; Vasiliev, J.M. Cytoskeletal Control of Fibroblast Length: Experiments with Linear Strips of Substrate. J Cell Sci 2001, 114, 4335-4341.

(5) Mũnoz, Z.; Shih, H.; Lin, C.C. Gelatin Hydrogels Formed by Orthogonal ThiolNorbornene Photochemistry for Cell Encapsulation. Biomater Sci 2014, 2, 1063-1072. 